\title{
Aktuelle Forschungsfelder der Wirtschaftsinformatik
}

\author{
DOI 10.1007/s11576-013-0401-3
}

\section{Die Autoren}

Prof. Dr. Martin Bichler ( $\varangle$ )

Decision Sciences \& Systems

Institut für Informatik

TU München

Boltzmannstraße 3

85748 München

Deutschland

bichler@in.tum.de

Prof. Dr. Thomas Hess

Institut für Wirtschaftsinformatik

und Neue Medien

Ludwig-Maximilians-Universität

München

Ludwigstraße 28

80539 München

Deutschland

thess@bwl.Imu.de

Prof. Ramayya Krishnan, Ph.D.

Management Science

and Information Systems

The Heinz College

Carnegie Mellon University

5000 Forbes Avenue

Pittsburgh, PA 15213-3890

USA

rk2x@cmu.edu

\section{Prof. Dr. Peter Loos}

Institut für Wirtschaftsinformatik im DFKI

Universität des Saarlandes

Stuhlsatzenhausweg 3

66123 Saarbrücken

Deutschland

loos@iwi.uni-sb.de

Online publiziert: 2014-01-04

This article is also available in English via http://www.springerlink.com and http://www.bise-journal.org: Bichler M, Hess T, Krishnan R, Loos P (2013) Research Areas in Business and Information Systems Engineering. Bus Inf Syst Eng. doi: 10.1007/s12599-0130309-x.

(C) Springer Fachmedien Wiesbaden 2014
„Für welche Themen steht die Wirtschaftsinformatik heute?“. „Welche Forschungsfragen prägen aktuell die Wirtschaftsinformatik?“. Diese Fragen werden öfter gerade von jüngeren Kollegen und Studierenden gestellt.

Die Zeitschrift WIRTSCHAFTSINFORMATIK (Business \& Information Systems Engineering) publiziert Forschungsergebnisse über den Entwurf, die Implementierung, und das Management von Informationssystemen, ebenso wie deren Auswirkung auf Individuen, Organisationen, die Wirtschaft und die Gesellschaft. Sie veröffentlicht Beiträge zu etablierten und neuen Forschungsfeldern an der Schnittstelle zwischen Betriebsund Volkswirtschaftslehre, Informatik und Informationstechnologie. Innerhalb dieses breiten Profils entwickelten sich über viele Jahre Teilcommunitys, die ihre eigenen Forschungsfragen und akzeptierte Methoden und Qualitätsstandards entwickelt haben. Nicht viele Kollegen haben eine Übersicht über die aktuellen Forschungsthemen in allen Teilcommunitys.

Ziel des Schwerpunktheftes ist die Publikation vorausschauender Artikel über Themen, die in der Wirtschaftsinformatik in den kommenden Jahren eine wichtige Rolle spielen werden. Fragestellungen der Wirtschaftsinformatik werden von technischen Entwicklungen beeinflusst. Es ist daher nicht überraschend, dass die Forschungsfragen in den letzten zwanzig Jahren auch einem Wandel unterworfen waren. Viele neue Forschungsfragen haben ihre Bedeutung durch die ubiquitäre Verfügbarkeit des Internets erlangt. Vergangenes Jahr haben der Fachbereich Wirtschaftsinformatik der Gesellschaft für Informatik e. V., die Wissenschaftliche Kommission Wirtschaftsinformatik im Verband der Hochschullehrer für Betriebswirtschaft e. V. und die Zeitschrift WIRTSCHAFTSINFORMATIK daher das vorliegende Schwerpunktheft initiiert, um eine Diskussion in der Forschungsgemeinschaft über aktuelle und neue Forschungsfelder der Wirtschaftsinformatik anzustoßen. Einerseits soll damit eine Übersicht über aktuelle Forschungsfelder in unserer Disziplin geschaffen werden. Andererseits sollen Anreize für Wissenschaftler gesetzt werden, um auch durch die Erstellung der Artikel in einen Diskurs über zukünftige Forschungsfelder zu treten. Für die meisten Einreichungen haben sich auch größere Gruppen von Autoren zusammengefunden.

Wir freuen uns sehr, dass der Aufruf zur Einreichung von Skizzen ein sehr positives Echo gefunden hat. 39 solcher Skizzen wurden an uns geschickt. Die Begutachtung über mehrere Runden wurde von einer Gruppe national und international renommierter Kollegen vorgenommen. Ein Teil der Autoren, deren Skizzen den im Aufruf gewünschten Kriterien entsprachen, wurde zur Einreichung von vollen Artikeln aufgefordert. In zwei weiteren Begutachtungsrunden wurde die Auswahl auf acht Artikel eingeschränkt, die in dieser Ausgabe der Zeitschrift veröffentlicht sind. Eine kleine Anzahl weiterer Artikel aus diesem Aufruf wird noch in späteren Ausgaben erscheinen. Die Artikel in der vorliegenden Ausgabe umfassen ein breites Spektrum an Themen:

„Informationssysteme für ,Wicked Problems' - Forschung an der Schnittstelle von Social Media und Collective Intelligence" von Schoder et al. behandelt Collective Intelligence. Das Forschungsfeld untersucht, wie über neue Formen der Interaktion neue Arten der Entscheidungsunterstützung entstehen. Das Thema hat in den vergangenen Jahren durch die Verbreitung von Plattformen wie YouTube, Twitter oder GitHub viel Aufmerksamkeit erhalten.

„Gestaltung der nächsten Inkarnation von Business-Intelligence - Flexibel gesteuerte Capability-Netzwerke zur Informationsintegration und -analyse" von Felden et al. beschreibt die nächste Stufe von Business-Intelligence-Anwendungen, in denen die Integration verteilter Daten und deren Auswertung Organisationen befähigen, schneller und gezielter auf Änderungen im Marktumfeld zu reagieren. Der Aufsatz basiert auf einer Analyse von über 1000 einschlägigen Veröffentlichungen.

„Modellbasierte Entscheidungsunterstützung in Produktions- und Dienstleistungsnetzwerken" von Mönch et al. beschreibt neue Forschungsthemen ebenfalls in einem etablierten Bereich der Wirtschaftsinformatik: den Entscheidungsunterstützungssystemen. Diese Themen umfassen die Integration verteilter Daten und Systeme, 
die Berücksichtigung autonomer Entscheidungsträger und von Informationsasymmetrien, die Modellierung von Präferenzen der Entscheider, effiziente und robuste Lösungsverfahren und effektive Methoden der Modellierung und Simulation.

„Energieinformatik - Aktuelle und zukünftige Forschungsschwerpunkte“ von Goebel et al. betrachtet Forschung (i) an energieeffizienten Systemen und (ii) an der Integration erneuerbarer Energien durch intelligente Distributionssysteme. Die Sicherung der Energieversorgung ist zweifellos eines der zentralen Themen unserer Gesellschaft und der Artikel beschreibt eine Reihe von Ansätzen wie die Wirtschaftsinformatik und angrenzende Disziplinen dazu beitragen können.

„Evolutionäre betriebliche Informationssysteme - Perspektiven und Herausforderungen einer neuen Generation von Informationssystemen" von Neumann et al. beschreibt Herausforderungen in der Entwicklung und Pflege großer Informationssysteme. Er diskutiert neue Konzepte aus der Softwaretechnik, neue Informationstechnologien und Möglichkeiten zur Berücksichtigung von Nutzeranforderungen und entsprechenden Adaption des Systems.

„Das Forschungsfeld ,Modellierung betrieblicher Informationssysteme - Gegenwärtige Herausforderungen und Eckpunkte einer zukünftigen Forschungsagenda" von Strecker et al. bietet eine Übersicht über neue Forschungsthemen in einem weiteren etablierten Feld der Wirtschaftsinformatik, der Modellierung von Informationssystemen. Der Artikel diskutiert neue Phänomene, die heute modelliert werden müssen, ebenso wie neue Modellierungssprachen und -werkzeuge.

„Geschäftsmodelle - Eine Forschungsagenda für die Wirtschaftsinformatik“ von Veit et al. ist ein verhältnismäßig neuer Themenbereich, der die grundlegenden Konzepte von Geschäftsmodellen untersucht. Die Autoren diskutieren Geschäftsmodelle in der IT-Industrie, Geschäftsmodelle, die durch IT ermöglicht werden, ebenso wie IT-Unterstützung zur Entwicklung von Geschäftsmodellen.

„User, Use \& Utility Research - Die Gestaltungsperspektive der Wirtschaftsinformatik auf den digitalen Nutzer" von Uebernickel et al. diskutiert Nutzerorientierung in der Wirtschaftsinformatik auf einer stärker strategischen Ebene. Die Autoren betonen die Auswirkung von Informationssystemen auf immer mehr Lebensbereiche sowie die Rolle, die Nutzer in der Gestaltung von Informationssystemen spielen können und sollen.

Natürlich kann ein Schwerpunktheft wie das vorliegende nicht alle relevanten Forschungsfelder der Wirtschaftsinformatik abdecken. Für einige etablierte Felder haben wir keine Einreichungen erhalten. Einige Autoren hatten eventuell nicht die Gelegenheit, einen Artikel einzureichen, manche Vorschläge mussten wir leider ablehnen, weil sie entweder zu eng waren oder die Kriterien im Aufruf zu Einreichungen nicht erfüllt haben. Die ausgewählten Beiträge unterstreichen aber sehr schön die Breite der Forschungsthemen in unserer Disziplin sowie den interdisziplinären Charakter, welche viele Fragen der Wirtschaftsinformatik kennzeichnet.

In einem breiten Fach wie der Wirtschaftsinformatik wird jeder Leser seine eigenen Favoriten haben, zu einigen mehr, zu anderen weniger Bezug haben. Insgesamt hat der Begutachtungsprozess aber zu einer interessanten Mischung an Beiträgen geführt, die einen guten Überblick über einige der wichtigen Themen in unserer Community bietet. Wir hoffen, dass die Artikel eine Grundlage für fruchtbare Diskussionen bieten und Ideen für zukünftige Forschungsprojekte liefern. Wir möchten uns bei den Gutachtern und den Autoren für ihr großes Engagement bedanken. Ebenso bedanken wir uns herzlich bei der Deutschen Bank, McKinsey\&Company und SAP für die weitere Unterstützung der Zeitschrift und wünschen Ihnen eine spannende Lektüre. 